\title{
Should we exercise caution with benzodiazepine use in patients with COPD?
}

\author{
Robert Rodriguez-Roisin ${ }^{1}$ and Judith Garcia-Aymerich²
}

\begin{abstract}
Affiliations: 'Servei de Pneumologia (Institut del Tòrax), Hospital Clínic, Institut d'Investigacions Biomédiques August Pi i Sunyer (IDIBAPS), CIBER Enfermedades Respiratorias (CIBERES), Universitat de Barcelona, Barcelona, Spain. ${ }^{2}$ Centre for Research in Environmental Epidemiology (CREAL), CIBERESP, Universitat Pompeu Fabra, Departament de Ciències Experimentals i de la Salut, Barcelona, Spain.
\end{abstract}

Correspondence: Robert Rodriguez-Roisin, Servei de Pneumologia (Institut del Tòrax), Hospital Clínic, Villarroel, 170, 08036-Barcelona, Catalonia, Spain. E-mail: rororodclinic.ub.es

0 @ERSpublications

Benzodiazepines should be used with caution in COPD patients http://ow.ly/wS51u

Benzodiazepines are agents that enhance the effect of the neurotransmitter $\gamma$-aminobutyric acid (GABA) on the GABAA receptor, resulting in sedative, hypnotic, sleep-inducing, anxiolytic, euphoric, anticonvulsant and muscle-relaxant properties [1]. These properties make benzodiazepines useful in managing anxiety, insomnia, agitation, seizures, muscle spasms and alcohol withdrawal, and as a pre-medication for medical procedures. Benzodiazepines are categorized as short-, intermediate- or long-acting. In general, benzodiazepines are safe and effective in the short term but the elderly are at an increased risk of suffering from both short- and long-term adverse effects.

The UK National Institute for Health and Clinical Excellence (NICE) highlights that opioids, benzodiazepines, tricyclic antidepressants and major tranquilisers are useful in palliating symptoms in patients with end-stage chronic obstructive pulmonary disease (COPD) [2]. Furthermore, all these agents, as well as oxygen therapy, should also be used when appropriate for breathlessness in patients with advanced COPD unresponsive to other medical therapy. However, American Thoracic Society/European Respiratory Society guidelines warn against the use of benzodiazepines and recommend avoiding hypnotics, if possible, in patients with severe COPD [3]. According to NICE and in the 2011 revision of the Global Initiative for Obstructive Lung Disease (GOLD) strategy [4], anxiety and depression, irrespective of whether they are considered separately or in combination, are viewed as major comorbidities in COPD and predict poor prognosis $[5,6]$. Both are often associated with younger age, female sex, cigarette smoking, more airflow limitation (lower forced expiratory volume in $1 \mathrm{~s}$ (FEV1)), cough, worse health-related quality of life and cardiovascular disease antecedents [7]. Furthermore, awareness of shortness of breath is higher in those patients with COPD who have symptoms of anxiety/depression [8]. Anxiety and depressive symptoms are very frequent in patients with COPD and appear to be more prevalent than in other chronic diseases [9-11]. Similarly, COPD patients with symptoms of anxiety/depression entering pulmonary rehabilitation have worse health-related quality of life using the COPD Assessment Test than asymptomatic patients [12]. Moreover, in the latter study, anxiety/depression were equally distributed across the new GOLD classification [4], which proposes a combined assessment taking symptoms (i.e. dyspnoea) and history of exacerbations, in addition to spirometry, into account.

Anxiety and depression symptoms may be confused with symptoms of COPD, so these psychiatric problems are often undiagnosed and untreated in clinical practice. In a cross-sectional primary care study, clinically relevant anxiety and/or depressive symptoms were observed in $18 \%$ and $42 \%$ of patients with COPD, respectively [13]. GOLD recommends that, in patients with COPD, both disorders should be treated according to usual guidelines, as there is no evidence that anxiety/depression should be treated differently in

Received: April 152014 | Accepted: April 162014

Conflict of interest: Disclosures can be found alongside the online version of this article at erj.ersjournals.com

Copyright @ERS 2014 
the presence of COPD [4]. In addition, COPD in patients with anxiety/depression should be treated as usual as there is no evidence that stable COPD should be treated differently in the presence of anxiety/depression.

In this issue of the European Respiratory Journal, Vozoris et al. [14] report that a third of a Canadian population of 177355 community-dwelling patients with a validated physician diagnosis of COPD, aged 66 years and older, was identified as new users of benzodiazepines. After propensity score matching, this cohort was well matched to an equivalent number of benzodiazepine nonusers. Compared with nonusers, new users were at significantly higher risk of outpatient exacerbations and emergency department visits for COPD or pneumonia. Although not significantly, the risk of hospitalisation for COPD or pneumonia was also elevated in new benzodiazepine users. Likewise, no significant differences between new users and nonusers of benzodiazepines were observed in regards to intensive care admissions during hospitalisations for COPD or pneumonia. Notwithstanding, all-cause mortality was slightly lower among new benzodiazepine users. The population of new users of benzodiazepines without exacerbations in the year prior to index date (namely, the date in which the incident use benzodiazepine started) had significantly higher risk for outpatient exacerbations, emergency department visits for COPD or pneumonia, hospitalisation for COPD or pneumonia and all-cause mortality. However, in the same subset of patients, there were no significant differences between new users and nonusers of benzodiazepines with respect to intensive care admissions for COPD or pneumonia. In contrast, in the subgroups of patients with one or more outpatient exacerbations and of those with one or more exacerbations requiring hospital admission, in the year prior to the index date, there were significant increases in the risk of outpatient exacerbations and emergency department visits for COPD or pneumonia among new users. It is noteworthy that, in the subset of patients with one or more exacerbation requiring presentation to hospital in the year prior to the index date, there was a significantly decreased risk of all-cause mortality among new benzodiazepine users.

These findings are of clinical interest and germane to the current management of COPD. They not only draw the attention of the respiratory community to the potential harmful effects of the use of benzodiazepines in advanced COPD regarding the different facets of lung function abnormalities but also to the evidence that the new use of benzodiazepines may be associated with increased risk of exacerbations and other life-threatening respiratory events. Benzodiazepines can be dispensed to patients with COPD for common and relevant important symptoms, such as dyspnoea, but also for the presence of anxiety/ depression. According to Vozoris et al. [14], these agents can be even more harmful in the more elderly and more-affected COPD patients. Thus, it could be likely that the incident use of benzodiazepines in older COPD patients, as presented in this Canadian study, could be at the origin of a vicious circle between the development (and recurrence) of exacerbations and the use of benzodiazepines. Accordingly, the (new) use of benzodiazepines in patients with COPD, more likely in the most advanced ones, could enhance the risk of increased exacerbations and other related life-threatening respiratory conditions.

Strengths of the study are the number of participants enrolled and the robust methodology applied to the data. That said, the results of this study should be cautiously interpreted in the light of its shortcomings, some of which have already been pointed out by the authors. Firstly, the retrospective recruitment of patients with COPD reinforces the concept that this kind of data is useful for generating new hypotheses but not for establishing solid conclusions, and always needs to be interpreted with some reservations. It is of note that in a very recent study of a Swedish cohort of more than 2000 severe COPD patients, the use of benzodiazepines and opioids in lower doses was not associated with increased admissions, suggesting that both medications might be safe for symptom reduction in advanced COPD [4, 15]. Secondly, the diagnosis of the events investigated, namely COPD exacerbations and pneumonia, can be confounded. We must recall that pneumonia is part of the differential diagnosis of COPD exacerbations [16]. Thirdly, and most importantly, estimates of the association are subject to a large degree of residual confounding. The authors used a sophisticated method to match new users and nonusers by propensity score [17], and succeeded in identifying two subgroups of COPD patients with similar distribution of measured confounders. Nonetheless, it has been reported that omission of important confounders in propensity score calculations not only leads to biased estimates of the treatment effect but also cannot be detected with model fit or discrimination analyses $[18,19]$. Indeed, no method can be of help when the relevant potential confounders are missing because they are not available in the original dataset. In the present study, the absence of data on relevant COPD outcomes, namely respiratory symptoms, symptoms of anxiety/depression, spirometric abnormalities, health-related quality of life, or smoking or work status, all of them potentially affecting both COPD prognosis and the duet of anxiety/depression, impairs the validity of the results $[6,20]$. The large differences in the estimates of the association between benzodiazepine use and all-cause mortality in patients without exacerbations in the previous year and those with hospital admissions for exacerbations clearly supports the notion that residual confounding can be present. Whether such a confounding scenario plays against or in favour of the role of new benzodiazepine use remains uncertain, as confounding of the assessment of drug effects in chronic diseases may easily produce paradoxical results [21]. In any case, a 
conservative interpretation could be that derived from the group with no previous exacerbations, as they are probably less affected by COPD severity and, accordingly, less subject to bias by residual confounding.

All in all, the findings of the study by VOzORIS et al. [14] highlight the relevance of customising comprehensive management to prevent exacerbations in patients with COPD, in particular hospitalisations, as well as targeting all those factors associated with anxiety/depression. Prevention of COPD exacerbations is as important as COPD treatment [4], so nowadays this is becoming one of the major priorities of the management of COPD. In this regard, physical activity limitation, use of long-term oxygen therapy, low body mass index, severe dyspnoea, low FEV1, poor quality of life, coexistence of other comorbidities, living alone, female sex, current smoking and/or poor socio-economic status [10], all outcomes associated with anxiety/depression, hence leading to benzodiazepine consumption, will need to be considered and identified. Then, if present, they will need to be adequately addressed and, if necessary, actively treated with wise therapeutic use of benzodiazepines. Let us hope that future research studies in this field will provide insights into all these reservations.

\section{References}

1 Shorter E. Benzodiazepines. In: A historical Dictionary of Psychiatry. London, Oxford Universtiy Press, 2005; pp. 41-42.

2 National Institute for Health and Clinical Excellence. Chronic obstructive pulmonary disease. www.nice.org.uk/ nicemedia/live/13029/49397/49397.pdf Date last accessed: April 15, 2014. Date last updated: June 2010.

3 Celli BR, MacNee W. Standards for the diagnosis and treatment of patients with COPD: a summary of the ATS/ERS position paper. Eur Respir J 2004; 23: 932-946.

4 Vestbo J, Hurd SS, Agusti AG, et al. Global strategy for the diagnosis, management, and prevention of chronic obstructive pulmonary disease: GOLD executive summary. Am J Respir Crit Care Med 2013; 187: 347-365.

5 Ng TP, Niti M, Tan WC, et al. Depressive symptoms and chronic obstructive pulmonary disease: effect on mortality, hospital readmission, symptom burden, functional status, and quality of life. Arch Intern Med 2007; 167: 60-67.

6 Eisner MD, Blanc PD, Yelin EH, et al. Influence of anxiety on health outcomes in COPD. Thorax 2010; 65: 229-234.

7 Hanania NA, Mullerova H, Locantore NW, et al. Determinants of depression in the ECLIPSE chronic obstructive pulmonary disease cohort. Am J Respir Crit Care Med 2011; 183: 604-611.

8 Neuman A, Gunnbjornsdottir M, Tunsater A, et al. Dyspnea in relation to symptoms of anxiety and depression: a prospective population study. Respir Med 2006; 100: 1843-1849.

9 Hill K, Geist RS, Goldstein RS, et al. Anxiety and depression in end-stage COPD. Eur Respir J 2008; 31: 667-677.

10 Maurer J, Rebbapragada V, Borson S, et al. Anxiety and depression in COPD: current understanding, unanswered questions, and research needs. Chest 2008; 134: Suppl., 43S-56S.

11 Coventry PA, Bower P, Keyworth C, et al. The effect of complex interventions on depression and anxiety in chronic obstructive pulmonary disease: systematic review and meta-analysis. PLoS One 2013; 8: e60532.

12 Hilmarsen CW, Wilke S, Engan $\mathrm{H}$, et al. Impact of symptoms of anxiety and depression on COPD Assessment Test scores. Eur Respir J 2014; 43: 898-900.

13 Yohannes AM, Baldwin RC, Connolly MJ. Depression and anxiety in elderly outpatients with chronic obstructive pulmonary disease: prevalence, and validation of the BASDEC screening questionnaire. Int J Geriatr Psychiatry 2000; 15: 1090-1096.

14 Vozoris NT, Fischer HD, Wang X, et al. Benzodiazepine drug use and adverse respiratory outcomes among older adults with COPD. Eur Respir J 2014; 44: 332-340.

15 Ekstrom MP, Bornefalk-Hermansson A, Abernethy AP, et al. Safety of benzodiazepines and opioids in very severe respiratory disease: national prospective study. BMJ 2014; 348: g445.

16 Rodriguez-Roisin R. Toward a consensus definition for COPD exacerbations. Chest 2000; 117: Suppl. 2, 398S-401S.

17 D’Agostino RB Jr, Propensity score methods for bias reduction in the comparison of a treatment to a nonrandomized control group. Stat Med 1998; 17: 2265-2281.

18 Weitzen S, Lapane KL, Toledano AY, et al. Principles for modeling propensity scores in medical research: a systematic literature review. Pharmacoepidemiol Drug Saf 2004; 13: 841-853.

19 Austin PC, Grootendorst P, Anderson GM. A comparison of the ability of different propensity score models to balance measured variables between treated and untreated subjects: a Monte Carlo study. Stat Med 1998; 26: 734-753.

20 Balcells E, Gea J, Ferrer J, et al. Factors affecting the relationship between psychological status and quality of life in COPD patients. Health Qual Life Outcomes 2010; 8: 108.

21 Garcia-Aymerich J, Marrades RM, Monso E, et al. Paradoxical results in the study of risk factors of chronic obstructive pulmonary disease (COPD) re-admission. Respir Med 2004; 98: 851-857. 